\title{
On the equivalence of a countable disjoint class of sets of positive measure and a weaker condition than total $\sigma-$ finiteness of measures
}

\section{Ficker}

Let $(X, S)$ be a measurable space and $S$ be a $\sigma$-algebra of subsets of $X$. A nonempty class $M$ is said to be a class of null sets if $M \subset S, M$ is closed under countable unions of sets and $E \cap F \in M$ whenever $E \in M$ and $F \in S$. It is possible to show that such concepts as absolute continuity, singularity and independence of measures can be studied simply by classes of null sets and that similar results can be obtained under the condition that each disjoint subclass of $S-M$ is countable, denoted $(S-M) C$. If $(X, S, \mu)$ is a measure space then $M=\{E \in S: \mu(E)=0\}$ is a class of null sets of $S$ and $S-M$ the class of all sets of positive measure. We say that a measure $\mu$ has the property $\sigma$ if there exists a sequence of totally finite measures $\left\{\mu_{n}\right\}_{n=1}^{\infty}$ on $S$ such that $\mu(E)=\sum_{n=1}^{\infty} \mu_{n}(E)$ for all $E \in S$. This property of measures is weaker than total $\sigma$-finiteness of measures. The main result of the present paper is as follows: Let $(X, S, \mu)$ be a measure space and $M=\{E \in S: \mu(E)=0\}$. Then $(S-M) C$ if and only if $\mu$ has the property $\sigma$.

1.

Throughout this paper $S$ is a $\sigma$-algebra of sets and $(X, S, \mu)$ is a measure space. We shall say a measure $\mu$ has the property $\sigma$, if there

Received 21 March 1969. Received by J. Austral. Math. Soc. 13 December 1968. Communicated by P.D. Finch. 
exists a sequence of totally finite measures $\left\{\mu_{n}\right\}_{n=1}^{\infty}$ defined on $S$ such that $\mu(E)=\sum_{n=1}^{\infty} \mu_{n}(E)$ for all $E \in S$. We note that each totally $\sigma$-finite measure $\mu$ has the property $\sigma$. The purpose of this paper is to prove the following Theorem.

THEOREM. Each class of disjoint sets of positive measure is countable if and only if $\mu$ has the property $\sigma$.

Next we give an example of a measure $\mu$ which is not $\sigma$-finite but has the property $\sigma$.

EXAMPLE. Let $X$ be a nonempty set. Suppose $S=\{\phi, X\}$, and $\mu(X)=+\infty, \mu(\phi)=0$. Put $\mu_{n}(X)=1, \mu_{n}(\phi)=0$ for $n=1,2,3, \ldots$, then $\mu(E)=\sum_{n=1}^{\infty} \mu_{n}(E)$ for all $E \in S$.

2.

Let $(X, S)$ be a measurable space. A nonempty class $N$ of sets, where $N \subset S$ is called a class of null sets of $S$

(i) if $E \in N$ and $F \in S$, then $E \cap F \in N$, and

(ii) if $E_{n} \in N, n=1,2,3, \ldots$, then $\bigcup_{n=1}^{\infty} E_{n} \in N$.

We note, if $(X, S, \mu)$ is a measure space, that the nonempty class $M$ of all those measurable sets $E$ for which $\mu(E)=0$ is a class of null sets of $S$ and $S-M$ is the class of all measurable sets $E$ of positive measure. The notation $(S-M) C$ indicates that each subclass of disjoint sets of $S-M$ is countable. The symmetric difference of two sets $E$ and $F$ is denoted by $E \Delta F$ and is defined by

$$
E \Delta F=(E-E) \cup(E-E)=\left(E \cap F^{C}\right) \cup\left(E \cap E^{c}\right) .
$$

The following Theorem is due to T. Neubrunn [4].

THEOREM 1. Let $(X, S)$ be a measurable space and $N$ be a class of nulZ sets of $S$. Suppose $(S-N) C$ and let $P$ be a property of measurable sets $E$, and be preserved under the formation of countable unions of disjoint sets. If at least one set $E$ in $S-N$ has the 
property $P$, then there exists a maximal set $M$ in $S-N$ with the property $P$ such that the conditions $E \in S, E$ has the property $P$, $E \subset M^{f} \quad i m p l y \quad E \in N$.

Let $(X, S, \mu)$ be a measure space. A set $E$ in $S$ is called an atom if $\mu(E)>0$, and if $F \in S$ such that $F \subset E$, then either we have $\mu(E-F)=0$ or $\mu(E)=0$.

LEMMA 1. Let $(X, S, \mu)$ be a measure space. If $E_{1}$ and $E_{2}$ are atoms, then either $\mu\left(E_{1} \Delta E_{2}\right)=0$ or $\mu\left(E_{1} \cap E_{2}\right)=0$. (cf. [1, p. 308]). All other concepts are used as in [3].

3.

LEMMA 2. Let $(X, S, \mu)$ be a measure space. If $\mu$ has the property $\sigma$ then $(S-M) C$.

Proof. Since $\mu$ has the property $\sigma$, there exists a sequence of totally finite measures $\left\{\mu_{n}\right\}_{n=1}^{\infty}$ such that $\mu(E)=\sum_{n=1}^{\infty} \mu_{n}(E)$, whenever $E \in S$. Then there exists a probability measure $\mu^{\prime}$ equivalent to $\mu$. It is sufficient to put $\mu^{\prime}(E)=\sum_{n=1}^{\infty} 2^{-n} \mu_{n}(E)\left[\mu_{n}(X)\right]^{-1}$, whenever $E \in S$. It is well known that $\left(S-M^{\prime}\right) C$. Since $\mu \equiv \mu^{\prime}$ i.e., $M=M^{\prime}$ we have $(S-M) C$.

COROLLARY. If $(X, S, \mu)$ is a measure space and $\mu$ has the property $\sigma$ then the class $A$ of all atoms in $S$ is countable.

Proof. According to Lemma 1 for any two sets $A, B \in A$ we have either $A \triangle B \in M$ and the set $A \cap B$ represents an atom or $A \cap B \in M$ and then the sets $A-B$ and $B-A$ represent two disjoint atoms i.e., two disjoint sets in $S-M$. Each class of disjoint sets in $S-M$ is countable according to Lemma 2 .

LEMMA 3. Let $(X, S, \mu)$ be a measure space. If there are no atoms in $S$ and $\mu$ is not $\sigma$-finite, then there exists in $S-M$ an uncountabie class of disjoint sets of finite measure.

Proof. There exists at least one set $E$ of positive and finite measure such that $E \subset X$ and $E \neq X$, since $X$ is not an atom. Now, 
suppose $(S-M) C$ and let $P$ be the following property of measurable sets G.

$$
\begin{aligned}
& P: G \text { is a countable union of disjoint sets of finite measure from } \\
& \quad S-M .
\end{aligned}
$$

There exists at least one measurable set with the above property, $E$ itself. Evidently the property $P$ is preserved under the formation of unions of countable disjoint sets. According to Theorem 1 there exists a maximal measurable set $M$ with property $P$ such that the conditions $E \in S, E \subset M^{f}$, imply $E \in M$. Otherwise $M^{f}$ would be an atom. Since $M$ is a countable union of disjoint sets of finite measure from $S-M$ and $X=M \cup M^{\mathcal{F}}$ then $\mu$ is a $\sigma$-finite measure. This is a contradiction.

LEMMA 4. Let $(X, S, \mu)$ be a measure space. If there are no atoms in $S$ and $\mu$ is not $\sigma$-finite, then $\mu$ has not the property $\sigma$.

Proof. According to Lemma 3 there exists an uncountable class of disjoint sets of positive and finite measure $\mu$, say $A=\left\{A_{t}, t \in T\right\}$. On the contrary let us assume that $\mu$ has the property $\sigma$, then $\mu\left(A_{t}\right)=\sum_{n=1}^{\infty} \mu_{n}\left(A_{t}\right)^{\prime}$ for $t \in T$. Then, there exists a positive integer $n_{0}$ such that $\mu_{n_{0}}\left(A_{t}\right)>0$ for an uncountable set $T^{\prime}$ of indices, where $t \in T^{\prime} \subset T$. This contradicts the total finiteness of the measure $\mu_{n_{0}}$. Thus $\mu$ has not the property $\sigma$.

REMARK 1 . We note, if $\mu$ has the property $\sigma$, then also $\mu_{E}$ has the property $\sigma$, where $\mu_{E}\left(F^{\prime}\right)=\mu\left(E \cap F^{\prime}\right)$, whenever $F \in S$.

LEMMA 5. Let $(X, S, \mu)$ be a measure space and let $(S-M) C$. Then $\mu$ has the property $\sigma$.

Proof. (i) Suppose, there are no atoms in $S$. Then we prove that $\mu$ is a $\sigma$-finite measure. On the contrary if $\mu$ is not $\sigma$-finite, then according to Lemma' 3 the class of all disjoint sets of positive measure $\mu$ is uncountable. This is a contradiction with $(S-M) C$. Therefore $\mu$ is a $\sigma$-finite measure, hence $\mu$ has the property $\sigma$. 
(ii) Let us admit that there are also atoms but of finite $\mu$-measure only. Then the class $A$ of all disjoint atoms is countable, i.e. $A=\left\{A_{1}, A_{2}, A_{3}, \ldots\right\}$. Put $A=\bigcup_{i=1}^{\infty} A_{i} A_{i} \in A, i=1,2,3, \ldots$, and put $\lambda(E)=\mu(F \cap A)$ and $\nu(F)=\mu\left(F \cap A^{c}\right)$, for all $E \in S$. Then $\lambda$ and $\nu$ are two $\sigma$-finite measures. Evidently $\mu=\lambda+\nu$ on $S$ and $\mu$ is a $\sigma$-finite measure, hence $\mu$ has the property $\sigma$.

(iii) Suppose now that there exist atoms $B$ such that $\mu(B)=+\infty$. The class of all such disjoint atoms is countable since $A \subset S-M$. Put $A=\bigcup_{i=1}^{\infty} A_{i}, A_{i} \in \mathrm{A}, i=1,2,3, \ldots$ Let us define

$$
\mu_{i}(E)= \begin{cases}1, & \text { if } \mu\left(E \cap A_{i}\right)=\mu\left(A_{i}\right), \\ 0, & \text { if } \mu\left(E \cap A_{i}\right) \neq \mu\left(A_{i}\right)\end{cases}
$$

whenever $E \in S$. We prove $\mu_{i}$ is a measure for each positive integer $i$. Fix $i$, then evidently $\mu_{i} \geq 0$ and $\mu_{i}(\phi)=0$. Let $\left\{E_{k}\right\}_{k=1}^{\infty}$ be a sequence of disjoint sets from $S$. Put $E=\bigcup_{k=1}^{\infty} E_{k}$. If

$\mu\left(E \cap A_{i}\right)=\mu\left(A_{i}\right)$ then $\mu_{i}(E)=1$. Then from $\mu\left(E \cap A_{i}\right)=\sum_{k=1}^{\infty} \mu\left(E_{k} \cap A_{i}\right)$ follows that $\sum_{k=1}^{\infty} \mu\left(E_{k} \cap A_{i}\right)=\mu\left(A_{i}\right)$. We prove that there is exactly one positive integer $k_{0}$ such that $\mu\left(E_{k_{0}} \cap A_{i}\right)=\mu\left(A_{i}\right)$. On the contrary suppose there are at least two such integers, say $k_{1}$ and $k_{2}$ then $\mu\left(E_{k_{1}} \cap A_{i}\right)>0$ and $\mu\left(E_{k_{2}} \cap A_{i}\right)>0$, since $E_{k_{1}} \cap E_{k_{2}}=\phi$ for $k_{1} \neq k_{2}$ and $E_{k_{1}} \cap A_{i} \subset A_{i}, E_{k_{2}} \cap A_{i} \subset A_{i}$. The latter two relations contradict the assumption that $A_{i}$ is an atom. Therefore $\mu_{i}\left(E_{k_{0}}\right)=1$, and $\mu_{i}\left(E_{k}\right)=0$ for all $k \neq k_{0}$. Then we have $\mu_{i}(E)=\sum_{k=1}^{\infty} \mu_{i}\left(E_{k}\right)$. Further $\mu_{i}(E)=0$, if $\mu\left(E \cap A_{i}\right) \neq \mu\left(A_{i}\right)$, then $\mu\left(E \cap A_{i}\right)=0$, since $\mu\left(A_{i}\right)=+\infty$ and $A_{i}$ is an atom. This implies $\mu\left(E_{k} \cap A_{i}\right)=0$ for $k=1,2,3, \ldots$, hence $\mu\left(E_{k} \cap A_{i}\right) \neq \mu\left(A_{i}\right)$ and we have $\mu_{i}\left(E_{k}\right)=0$ for $k=1,2,3, \ldots$, this implies $\mu_{i}(E)=\sum_{k=1}^{\infty} \mu_{i}\left(E_{k}\right)$. 
Therefore $\mu_{i}$ is a $\sigma$-additive set function, hence $\mu_{i}$ is a measure.

Let $n$ be a positive integer, put $\nu_{n}=\sum_{i=1}^{n} \mu_{i}$. Evidently $\nu_{n}$ is a totally finite measure on $S$. Now we prove $\mu(E \cap A)=\sum_{n=1}^{\infty} \nu_{n}(E)$, whenever $E \in S$. Evidently $\mu(E \cap A)=\sum_{i=1}^{\infty} \mu\left(E \cap A_{i}\right)$. If $E \in S$ is such a set that there exists at least one index $i_{0}$ that $\mu\left(E \cap A_{i_{0}}\right)=\mu\left(A_{i_{0}}\right)$, then $\mu(E \cap A)=+\infty$ and then for all $n \geqq i_{0}$ we have $\nu_{n}(E) \geqq 1$, therefore $\mu(E \cap A)=\sum_{n=1}^{\infty} \nu_{n}(E)$. If $\mu\left(E \cap A_{i}\right) \neq \mu\left(A_{i}\right)$ for $i=1,2,3, \ldots$, then $\mu\left(E \cap A_{i}\right)=0$ for all positive integers $i$ and also $\mu_{i}(E)=0$. The latter condition implies $\nu_{n}(E)=0$, for $n=1,2,3, \ldots$, therefore $\mu(E \cap A)=\sum_{n=1}^{\infty} \nu_{n}(E)$. Hence $\mu_{A}$ has the property $\sigma$.

Let $E \in S$, then $\mu(E)=\nu(E)+\lambda(E)$, where $\nu(E)=\mu(E \cap A)$ and $\lambda(E)=\mu\left(E \cap A^{C}\right)$. Then $\lambda$ is a $\sigma$-finite measure, since it is either (i) or (ii), therefore $\lambda(E)=\sum_{n=1}^{\infty} \lambda_{n}(E)$.

Finally, if $\mu_{2 n-1}(E)=\nu_{n}(E)$ and $\mu_{2 n}(E)=\lambda_{n}(E)$, for $n=1,2,3, \ldots$, whenever $E \in S$ then $\mu(E)=\sum_{n=1}^{\infty} \mu_{n}(E)$. This completes the proof.

Now from Lemmas 2 and 5 immediately follows,

THEOREM 2. Let $(X, S, \mu)$ be a measure space. Then $(S-M) C$ if and only if $\mu$ has the property $\sigma$.

\section{References}

[1] Nelson Dunford and Jacob T. Schwartz, Linear operators, Part I (Interscience Publishers, New York, London, 1958).

[2] V. Ficker, "Dominated classes and related questions", Acta Fac. Remum Natur. Univ. Comenian. 10, 7 (1966), 3-18.

[3] Paul R. Halmos, Measure theory, (Van Nostrand, New York, 1950). 
[4] Tibor Neubrunn, "Remark on absolute continuity of measures" (Russian) Mat.-Fyz. Casopis Sloven. Akad. Vied. 16 (1966), 21-30.

Department of Mathematics, University of Newcastle, New South Wales. 